\title{
A Couple of Generator and Motor as Electric Transmission System of a Driving Shaft to Long Distance Driven Shaft
}

\author{
Iswanjono $^{1}$, Y.B. Lukiyanto ${ }^{2, *}$, Budi Setyahandana ${ }^{2}$ and Rines ${ }^{2}$ \\ ${ }^{1}$ Electrical Engineering, Sanata Dharma University, Mrican, Tromol Pos 29, Yogyakarta, Indonesia \\ ${ }^{2}$ Mechanical Engineering, Sanata Dharma University, Mrican, Tromol Pos 29, Yogyakarta, Indonesia
}

\begin{abstract}
Wind energy converter commonly converts wind power to shaft and electric power. One of the problems of transmitting shaft power is the limited distance between the driving to the driven shaft. This paper describes an experimental study of electrical transmission from driving to the driven shaft that was carried out on three modes of cable lines transmission. The driving shaft was attached to an electric generator that converts shaft $r$ to electric power. The driven shaft was coupled by electric motor which reconverts the electric to shaft power. The generator and motor were BLDC and BDC permanent magnet electric machines. The distance of the driving to the driven shaft was 300 meters. The electric power was transmitted using wires. The wires consisted of 2 and 3 cables commonly used by peoples and sold in market place. The results showed that electric transmission mode on the experiment has important role on performance of shaft power to electric power conversion on driving generator and energy losses on cables transmission. DC on small wires electric transmission gave total efficiency of $16.2 \%$. DC on large wires electric transmission gave total efficiency of $32.9 \%$. AC on large wires electric transmission gave total efficiency of $36.4 \%$.
\end{abstract}

\section{Introduction}

Wind energy is one of the most popular renewable energy resources for remote area to be converted into mechanical and electrical energy. Wind pump is one application of wind energy conversion to mechanical energy. The wind pump can be used for water pumping system with head less than 3 meter up to more than 30 meter [1] also for water desalination system [2,3]. Dependable standalone pumping system for irrigation became a favourite solution for the farmer [4] and for remotely located inhabitants that are not connected to national power grid system [5].

As water pumping system with wind energy play an important role to reduce the consumption of conventional energy sources [6], the reseach development with wind energy source water pumping system have been specific attentions to researchers. The excitation capacitor for a wind pump using induction motor coupled to a centrifugal pump was investigated to find an optimal excitation capacitor for a given wind rotor speed [7]. Alternative energy storage for wind power was analyzed for Columbia River Gorge area [8]. The conclusion was that the economic and technical criteria are the most important ones for decision makers. A pumping system compound of a wind turbine, a selfexcited induction generator (SEIG), an induction motor (IM), and a centrifugal pump (CP), which aims to ensure the water pumping in optimum conditions was studied
[9]. The result was that the maximization strategy MPPT on the static converter SCV is effective in the search of the maximum power where the wind speeds vary in large portions.

This experiment studied energy losses on wind pump with long-shaft power transmission. Driving shaft power from wind turbine was converted to electrical power by electric generator. The electrical power was then transmitted by 300 meter wires length to electric motor. The electric power was then converted to mechanical driven shaft power. The driven shaft power was coupled with a centrifugal reaction pump. Detail investigation was important to find out energy losses of every component. Then, the final investigation found the efficiency of every component and the total efficiency of the transmission system.

\section{Experiment Methods}

\subsection{Equipments}

The experiment equipments consisted of a transmission unit as the main unit (Fig. 1), a driving unit and a driven unit as supporting units. Each unit has specific equipments. The main equipments of main unit comprised of an electric generator, diodes, 300 meters of cables and an electric motor. The electric generator was 350 watt permanent magnet brushless DC (BLDC)

Corresponding author: lukiyanto@usd.ac.id 
generator. The BLDC generator was usually used for driving motor of electric bikes. The output of the generator were three phases of alternating current. The alternating current $(\mathrm{AC})$ then was converted into direct current (DC) by diodes circuits. The cables was used to transmit electric power from electric generator on DC or $\mathrm{AC}$ mode to the electric motor. On the DC mode, the diodes circuit was installed between electric generator and the cables. On the AC mode, the diodes circuit was installed between cables and the electric motor. The electic motor as a driven motor was 250 watt permanent magnet brushed DC (BDC) motor. The BDC motor was also usually used for driving motor of electric bikes.

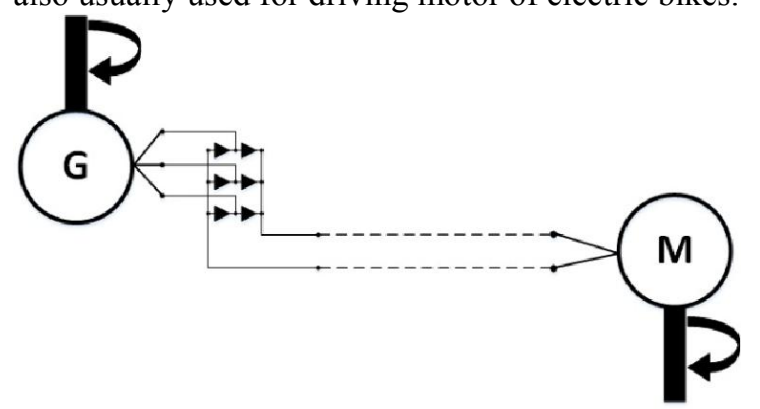

Fig. 1. Schematics of Transmission Unit

The supporting units as secondary equipments comprised of driving motor, driving transmission, driving shaft, driven shaft and driven pump. Driving motor, driving transmission and driving shaft were functioned as a driving unit to regulate shaft power input of electric generator (Fig. 2). The driving motor was 3 phase motor of 3000 watt. The speed of driving motor was adjusted by transistor inverter of $3 \mathrm{PH}-220 / 240 \mathrm{~V}$ $5.5 \mathrm{~kW}$. The driving transmission used to transmit mechanical power was belt and pulleys to decrease the shaft speed. Three and seven inches pulley diameter were connected by a A-52 belt and attched to shaft driving motor and shaft generator. The driving motor and generator were attached on a rigid structure. The distance of the generator mounting could be adjusted to maintain belt tension.

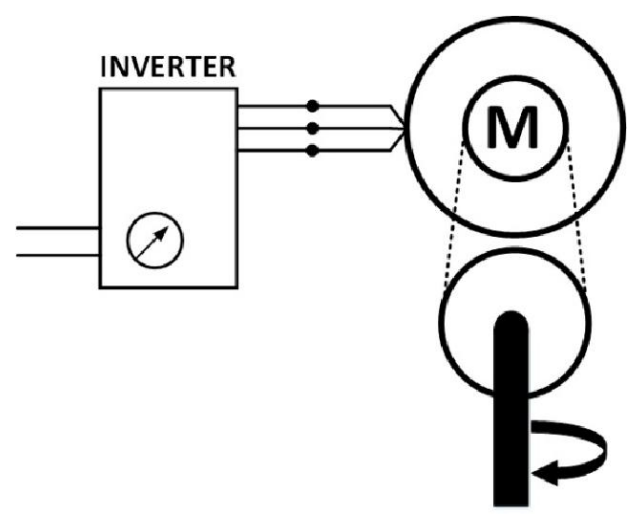

Fig. 2. Schematics of Driving Unit

The driven pump (Fig. 3) was a centrifugal reaction pump [10]. The pump was arranged from two horizontal pipes as impeller and one vertical pipes as inlet. All pipes has diameter of $5 \mathrm{~cm}$. The pipes were configured on $\mathrm{T}$ shape junction. The total diameter of the pump was
$140 \mathrm{~cm}$. The pipes outlet diameter was $2 \mathrm{~cm}$ and configured on $\mathrm{S}$ shape configuration in lined with the pump impeler. The pump head were maintained constant at $150 \mathrm{~cm}$ and measured from water surface to the center of pipes outlet. The driven shaft was attached on a rigid structure by two bearings. The main dimensions of the structure were $150 \mathrm{~cm}$ wide, $150 \mathrm{~cm}$ length and $200 \mathrm{~cm}$ height. Upper side of the structure was shrouded with a hole on the bottom. The water from outlet pipes was collected on the shroud and then its flow rate was measured. At the bottom of the structure an adjustable main pond was installed to maintain the water surface at a constant high level.

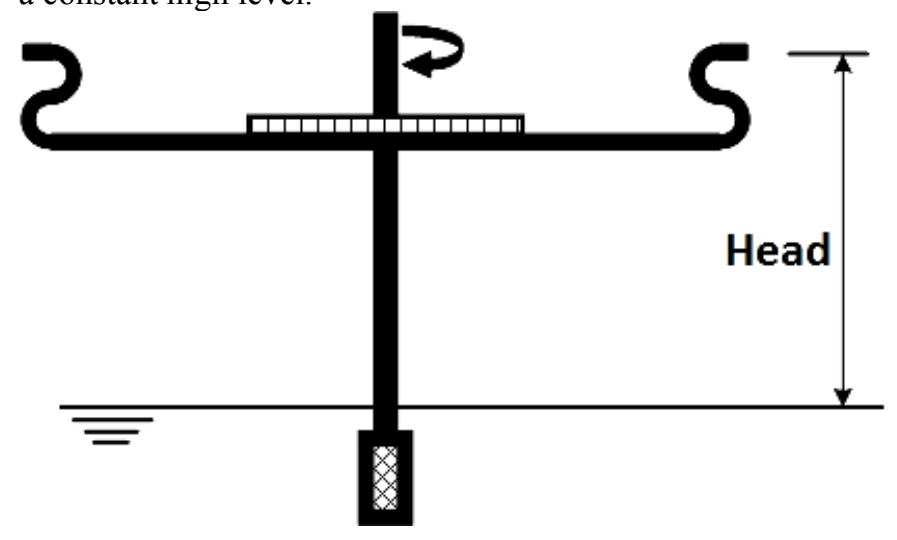

Fig. 3. Schematics of Driven Unit

\subsection{Variations and Parameters}

The experiments consisted of three variations depend on cable diameter and position of diodes circuits. First variation was DC on small wires (Fig. 1). On this variation, diodes circuits were installed after generator. Electric power was transmitted on DC mode by two cables. Dimension of the small cables were $2 \times 1.5 \mathrm{~mm}$ with 300 meter length. Second variation was DC on large wires. On this variation, diodes circuits were installed similar with first variation and electric power was also transmitted on DC mode. Dimension of the large cables were $2 \times 10 \mathrm{~mm}$ with 300 meter length. The third variation was AC on large wires. On this variation, diodes circuits were installed before driven motor. Electric power was transmitted on AC mode by three cables.

Torque-meters was installed on both of BLDC generator and BDC motor to measure driving and driven shaft powers, $W_{\text {shaft generator and }} W_{\text {shaft motor }}$. The shaft power $W_{\text {shaft }}$ was calculated with equation : Where :

$$
W_{\text {shaft }}=2 \pi \dot{n} F r
$$

$$
\begin{aligned}
& n=\text { shaft speed, rps (rotation per second) } \\
& F=\text { force at the end of arm torque (Newton) } \\
& r=\text { radius of arm torque (meter) }
\end{aligned}
$$

Radius or arm torque were constant of $15 \mathrm{~cm}$ for driving shaft and of $39 \mathrm{~cm}$ for driven shaft.

Ampere-meters and volt-meters were also installed on the ouput of BLDC generator and on the input to $\mathrm{BDC}$ motor to measure electrical powers, and. Electrical 
power output of BLDC generator, Welectrical generator was the input power to cables transmission. Electrical power input to BDC motor, Wactricalmotor was the output power of cables transmission.

Efficiencies of driving generator, transmission and driven motor were :

$$
\begin{aligned}
\eta_{\text {generator }} & =\frac{\text { Welectical generator }_{\text {shaft generator }}}{\eta_{\text {transmission }}}=\frac{\text { Wlectrical motor }_{\text {electrical generator }}}{W_{\text {motor }}}=\frac{w_{\text {shaft motor }}}{W_{\text {electrical motor }}}
\end{aligned}
$$

To start measurement, the potensiometer on the inverter was turned slowly until the pump was pumped the water. Data measurement was recorded when the system on steady state condition which was showed by the pumping water flow rate constant. Other data measurements was recorded on the other different generator shaft speed. The maximum shaft speed of the BLDC generator and of the pump were limited on 650 rpm $155 \mathrm{rpm}$. Other limitation were maximum power of BLDC generator (385 watt) and BDC motor ( 275 watt). The maximum power was limitated about 10 percent of rated power.

\section{Results and Discussions}

Based on collected data measurements, the maximum shaft speed of BLDC generator and BDC motor were $621 \mathrm{rpm}$ and $154 \mathrm{rpm}$. The both of maximum speeds were still below the allowed maximum speed. The maximum power of BLDC generator and BDC motor were 368.42 watt and 101.80 watt. The both of maximum powers were also still below the allowed maximum rated power. The minimum shaft speed of BLDC generator and BDC motor were 140 and $84 \mathrm{rpm}$. The generator and motor could operated below these minimum speed but the pump only rotate without pumping the water. The centrifugal reaction pump was start pumping water when the driven shaft speed round about $82 \mathrm{rpm}$.

The data measurements were then processed to show the performance of system with 3 variations. Figure 4, Figure 5 and Figure 6 showed the performance of BLDC generator and $\mathrm{BDC}$ motor in $\mathrm{DC}$ on small wires mode, $\mathrm{DC}$ on large wires mode and $\mathrm{AC}$ on large wires mode.

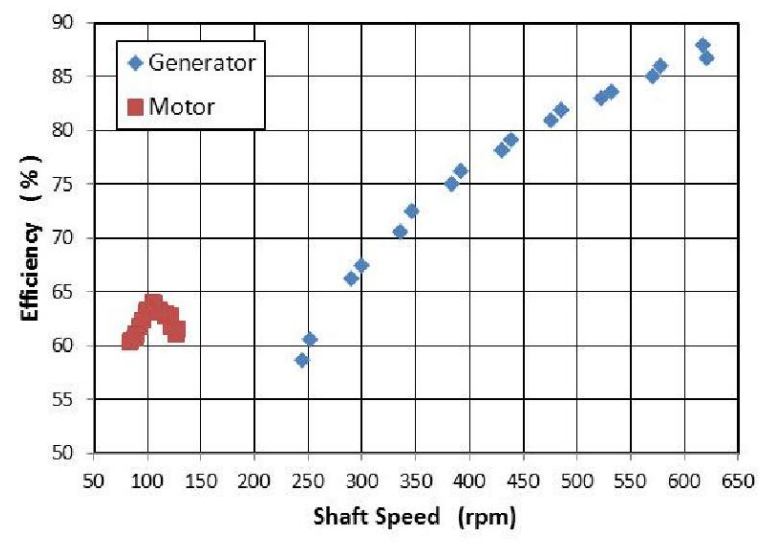

Fig. 4. DC on small wires mode

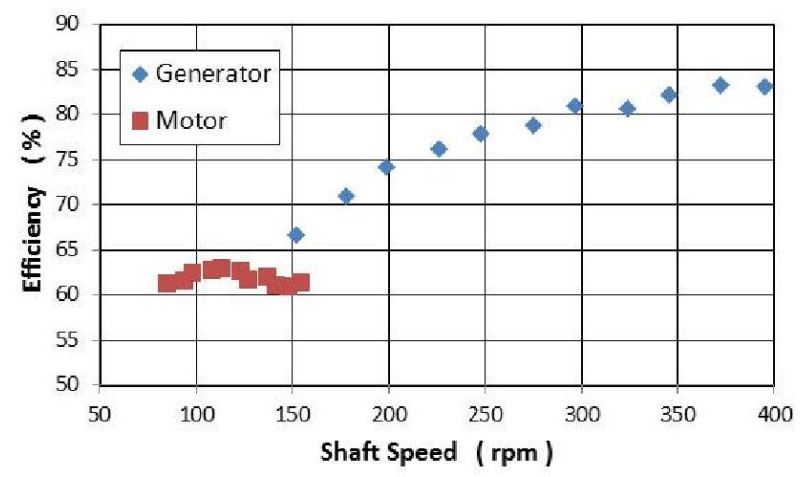

Fig. 5. DC on large wires mode

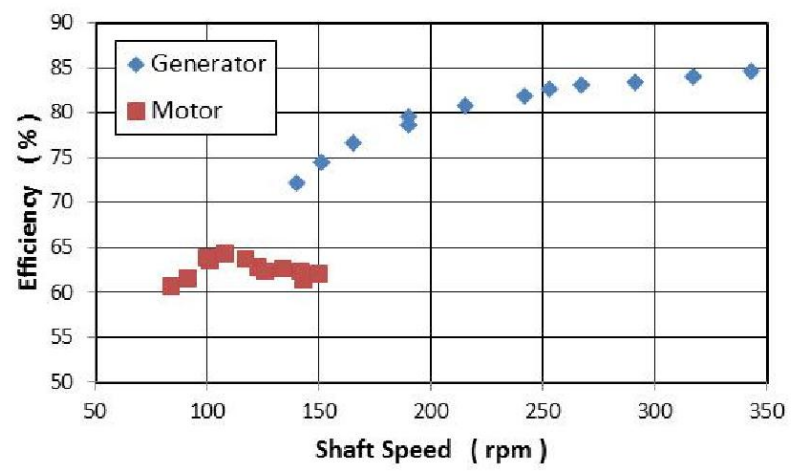

Fig. 6. AC on large wires mode

In Fig. 4, Fig. 5 and Fig. 6, the trend lines of electric generator efficiency have similarities. The efficiency of generator increased when the shaft speed increased. The characteristic of BLDC generator was similar with [11]. The higher the shaft speed, the higher the performance of generator from electric motor bike. On a particular shaft speed, the performance of the generator could have different values for different loads.

In Fig. 4, Fig. 5 and Fig. 6, the driving motor efficiency of the three variation modes has similar value between $60 \%$ up to $65 \%$. On these variations, the driven motor has similar characteristic load of the centrifugal reaction pump that operates between $82 \mathrm{rpm}$ up to $155 \mathrm{rpm}$ as maximum shaft speed of the pump on 150 meter head. On the variation of DC on Small Wires mode, the maximum driven motor shaft speed was only 
$128 \mathrm{rpm}$, lower than two other variation modes. This was because on this mode, when the driven motor shaft speed was at $128 \mathrm{rpm}$, the driving generator shaft speed and power reached allowed limited values.

On the variation of DC on Small Wires mode (Fig.4), the range driving generator and driven motor shaft speed was $377 \mathrm{rpm}$ and $44 \mathrm{rpm}$ or on range ratio of $8.6: 1$. On the variation of DC on Large Wires mode (Fig.5), the range driving generator and driven motor shaft speed was $243 \mathrm{rpm}$ and $69 \mathrm{rpm}$ or on range ratio of $3.5: 1$. On the variation of DC on Large Wires mode (Fig.6), the range driving generator and driven motor shaft speed was $203 \mathrm{rpm}$ and $66 \mathrm{rpm}$ or on range ratio of $3.1: 1$. Based on the range ratio values, on the variation of DC on Small Wires mode was needed largest driving generator shaft power than the two other modes. The variation of $\mathrm{AC}$ on Large Wires mode was smallest driving generator shaft power. The performance of the driven motor shaft power on the three modes was similar. The power losses of DC on Small Wires mode was the largest, while the power losses of AC on Large Wires mode was the smallest on this experiment.

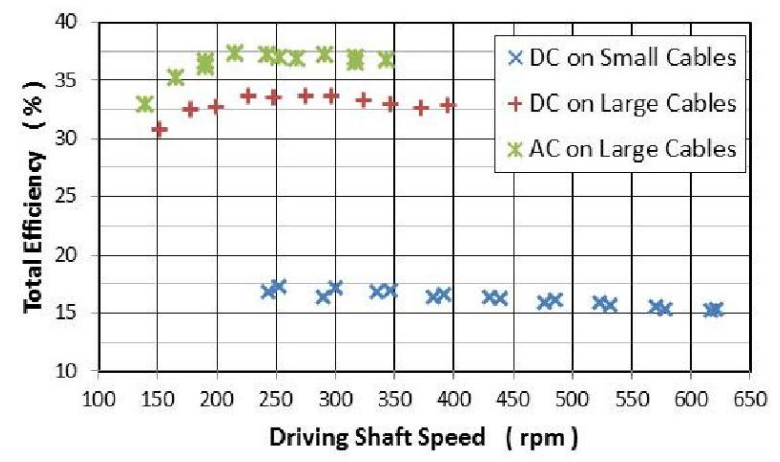

Fig. 7. Total efficiency based on driving shaft speed

In other word, total efficiency energy transmitted from driving shaft to the driven shaft of AC on Large Wires mode has highest efficiency value than the two other modes (Fig. 7). Total efficiency of DC on Small Wires mode has smallest efficiency value. The average efficiencies of DC on Small Wires mode, of DC on Large Wires mode and of AC on Large Wires mode were $16,2 \%, 32,9 \%$ and $36,4 \%$ respectively. These values was lower compare to [12] but has less equipment on the system.

\section{Conclussions}

A long shaft to shaft electrically transmission on three variation was tested and evaluated. The energy conversion of shaft power to electric power, the electric transmission in three variations and energy conversion of electric power to shaft power conversion prosesses was possible to find total efficiency of each variations. The centrifugal reaction pump was also satisfied as the windpump load with wide range shaft speed.

Electric transmission mode on the experiment has important role on performance of shaft power to electric power conversion on driving generator and energy losses on cables transmission. DC on small wires electric tranmission gave total efficiency of $16.2 \%$. DC on large wires electric tranmission gave total efficiency of 32.9 $\%$. AC on large wires electric tranmission gave total efficiency of $36.4 \%$. Since a considerable amount of energy losses during the energy conversion and transmission prosesses, economical aspects should be considered to avoid such losses in wind pumping system.

\section{References}

1. P.T. Smulders, Energy for Sustainable Development, 2.5., p.8-13, (1996)

2. E.S. Hrayshat, International Journal of Green Energy, 4:5, p.471-481, (2007)

3. G. Xenarios, P. Papadopoulos, E. Tzen, Desalination and Water Treatment, 51:4-6, p. 12191228, (2013):

4. B.S. Saravana, V. Rajini, A.Paramasivam, IERI Procedia, 5, p. 153 - 160, (2013)

5. S. Rehman, A.Z. Sahin, Renewable and Sustainable Energy Reviews, 16, p. 4470-4478, (2012)

6. C. Gopal, M. Mohanraj, P. Chandramohan, P. Chandrasekar, Renewable and Sustainable Energy Reviews, 25,p. 351-370, (2013)

7. M. Chaabene, M.B.A. Kamoun, M. Ouali, Smart Grid and Renewable Energy, 2.2., p.116 (2011)

8. T.T. Dain, D. Lim, F.A. Gomez, J. Schwarz, A. Jonanovic, Int.J. Sustainable Energy, p.1-15 (2011))

9. T. Ouchbel, , S. Zouggar, M.L. Elhafyani, M. Seddik, M. Oukili, A. Aziz, F.Z. Kadda, Energy Conversion and Management, 78, p. 976-984, (2014)

10. B. Setyahandana, Y.B.Lukiyanto, Rines, Proceeding of the 15 International Conference on QIR (Quality in Research), ISSN 1411-1284, p.324-332, (2017)

11. Y.B.Lukiyanto, Advanced Materials Research, 789, p. 443-448, (2013)

12. D.D. Lara, G.G. Merino, B.J. Pavez, J.A. Tapia, Energy Conversion and Management, 52, p. 795803, (2011) 